\title{
Does Droplet Infection Play a Role in the Transmission of Leprosy?
}

\author{
J. C. PEDLEY AND JOHN G. GEATER
}

\begin{abstract}
Experiments are described aimed at repeating in the context of modern knowledge the long neglected work of Schaffer on the release of Mycobacterium leprae from the upper respiratory passages during sneezing, coughing and normal speech. In two Bhutanese patients large numbers of $M$. leprae, including some of normal morphology, were projected during sneezing up to a distance of $30 \mathrm{~cm}$ from the face and smaller numbers to a distance of $50 \mathrm{~cm}$. None could be recovered during $20 \mathrm{~min}$ of normal speech. Only patients with untreated or relapsing lepromatous leprosy are implicated, as distinct from borderline cases, the criterion being the presence of a highly bacilliferous nasal discharge.
\end{abstract}

\section{Does Droplet Infection Play a Role in the Transmission of Leprosy?}

At the first International Leprosy Congress at Berlin, Schäffer (1898) described experiments in which sets of microscope slides were placed at distances up to $50 \mathrm{~cm}$ in front of 2 patients with severe lepromatous leprosy during the process of talking, coughing and sneezing. Large numbers of acid-fast bacilli were found on the slides. At that time it was impossible to identify these as Mycobacterium leprae, and Schäffer's work suffered from the neglect which for so many years surrounded nasal infection in leprosy, on the grounds that authentic $M$. leprae could be confused with contaminating acid-fast bacilli at this site.

The attention drawn by one of us to nose blows (Pedley, 1973) once again stimulated interest in Schäffer's findings. His original paper was written in German. Dr R. J. W. Rees arranged for its translation into English by Dr Bodingius, and the relevant sections are as follows.

" 20-30 glass slides, closely adjacent, were placed in front of the patient. The patient talked for $10 \mathrm{~min}$, reading or counting, after which time the slides had on them specks of mucus of different sizes. The slides were left till dry, fixed over a flame, and stained by the Ziehl-Neelsen method. For counting slides were divided into 8 quadrants, numbers of acid-fast bacilli in each quadrant were counted using oil immersion lens, and the total number of bacilli then calculated. Mistakes were avoided by decolourising with acid alcohol, bacilli about which there could be no doubt showing characteristic cigar shaped bundles and the occurrence of conglomerates (globi). No bacillary complexes were counted, only single organisms. It was found that if the patient stood upright, droplets were projected as far as $30-50 \mathrm{~cm}$ onto surfaces held in front of the patient. 
Results. One patient released 10,000 to 25,000 bacilli. The other patient released 75,000 to 120,000 bacilli and on one occasion 185,000 bacilli. In both patients investigated the secretion from the nose contained very many bacilli. Extremely large numbers of bacilli were released in sneezing (patients were given sneezing powder) ... in one sneeze a patient released 110,000 bacilli.

Conclusions. Leprosy patients with mucous membrane affection of the respiratory tract . . release thousands of bacilli during speech, coughing and sneezing. Are the released bacilli viable? Some authors think they are dead. This is still unproved because no culture or animal inoculation has been possible up till now. We should not worry too much because clinical experience has shown that the danger of leprosy transmission is in fact extremely small. Cases of close contact for years do not acquire leprosy. Bacilli in great masses reach healthy persons without leading to disease. We may however not conclude that bacilli released by the respiratory tract do not play a role in the transmission of leprosy."

Nowadays patients with untreated florid lepromatous leprosy are not easy to find, but it seemed to us worthwhile to repeat Schäffer's work against the background of modern knowledge. An opportunity arose when a suitable patient attended at Mongar Hospital, Bhutan. The patient, a Bhutanese patient about 35 years of age, had been suffering from lepromatous leprosy for 5 years and was untreated. The disease was well advanced, and smears of the noseblow showed that the mucus was heavily infected with acid-fast bacilli and numerous globi. The B.I. of skin smears was 5.0.

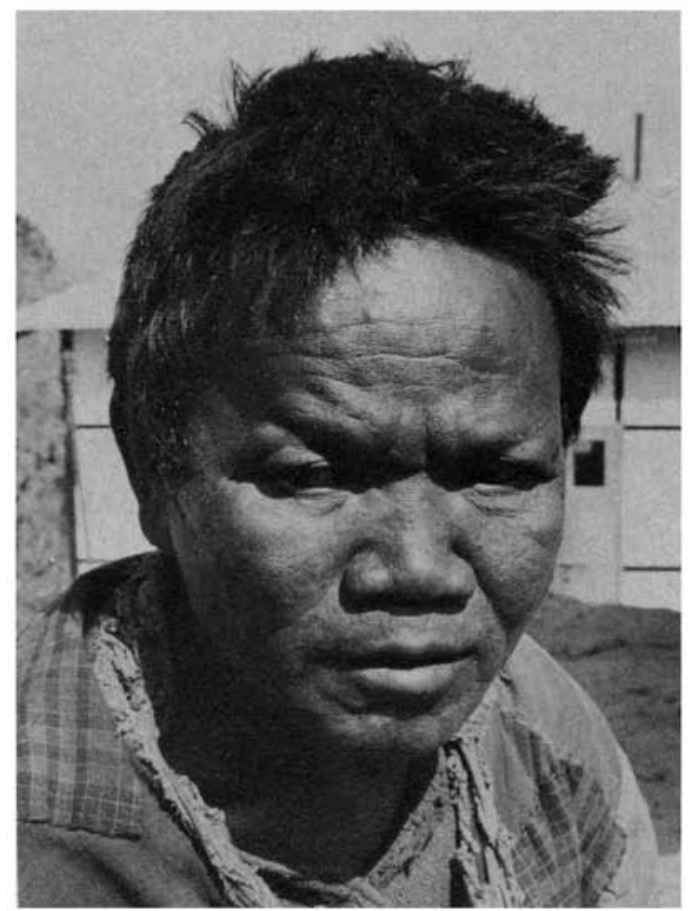

Fig. 1. Name: Sonam. Nationality: Bhutanese. Age: 35. Untreated lepromatous leprosy 5 years. 


\section{Method}

Sneezing was induced by tickling the nasal mucosa. The patient directed his sneeze towards microscope slides placed at distances of 20,30 and $50 \mathrm{~cm}$ from the face. Slides were fixed, stained by the Ziehl-Neelsen method and examined for acid-fast bacilli.

\section{Findings}

\section{SNEEZE AT $20 \mathrm{CM}$}

Two slides with a total area of approximately $39 \mathrm{~cm}^{2}$ were examined systematically and revealed the presence of 500 droplets of sizes varying from $0.2 \mathrm{~mm}$ to $0.7 \mathrm{~mm}$ in diameter. Fifty droplets (i.e. $10 \%$ of the total) were examined in detail in succession as they occurred on the slides using the oil immersion lens. In 35 of the 50 droplets (i.e. $75 \%$ ) bacilli were found, arranged as follows:

$$
\begin{array}{ll}
\text { Globi } & 20 \\
\text { Smaller aggregates (cigar } & \\
\quad \text { shaped bundles, clusters, etc.) } & 32 \\
\text { Single bacilli } & 50-100
\end{array}
$$

Figure 2 shows a single field from a slide at this distance.

SNEEZE AT $30 \mathrm{CM}$

Three slides from which a total area of approximately $35 \mathrm{~cm}^{2}$ was systematically searched revealed the presence of about 340 droplets with the same variation in size as recorded above. Fifty droplets were examined under oil immersion. Thirteen showed the presence of acid-fast bacilli, classified as follows:

$\begin{array}{lr}\text { Globi } & 8 \\ \text { Smaller aggregates } & 16 \\ \text { Single bacilli } & 20\end{array}$

Figure 3 shows a single field from a droplet which was $0.4 \mathrm{~mm}$ in diameter at this distance.

\section{SNEEZE AT $50 \mathrm{CM}$}

A second patient, a Bhutanese youth aged 22 years, suffering from untreated and well advanced lepromatous leprosy (B.I. of skin 3.5, B.I. of nasal mucus secretion 4.0) sneezed towards a slide held at $50 \mathrm{~cm}$ from the face. A search of the slide revealed the presence of 7 droplets, in 6 of which acid-fast bacilli were found, occurring singly, in groups and in globi.

At all distances individual bacilli were encountered which preserved the stain uniformly.

Figure 4 shows a single field from a slide at $50 \mathrm{~cm}$.

The photomicrographs from both patients are filed at the Leprosy Study Centre, London.

Further experiments were undertaken with the first of these two patients in order to ascertain the release of bacilli in talking, snoring (simulated with the mouth open), and panting after vigorous exercise. Table 1 summarizes the findings in all these experiments. 


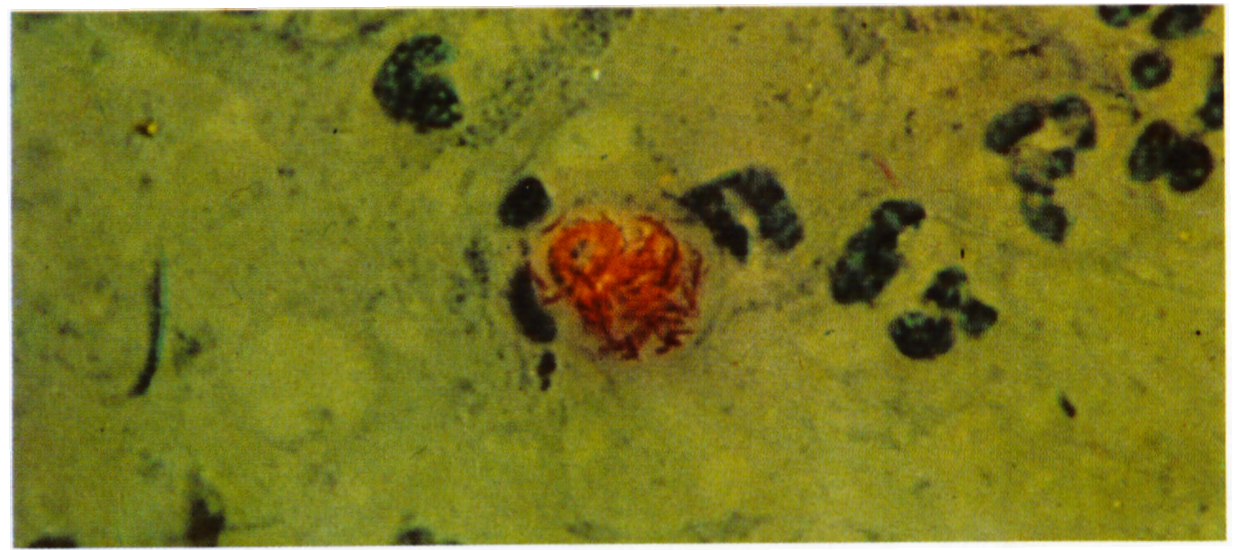

Fig. 2. Sneeze at $20 \mathrm{~cm}$.

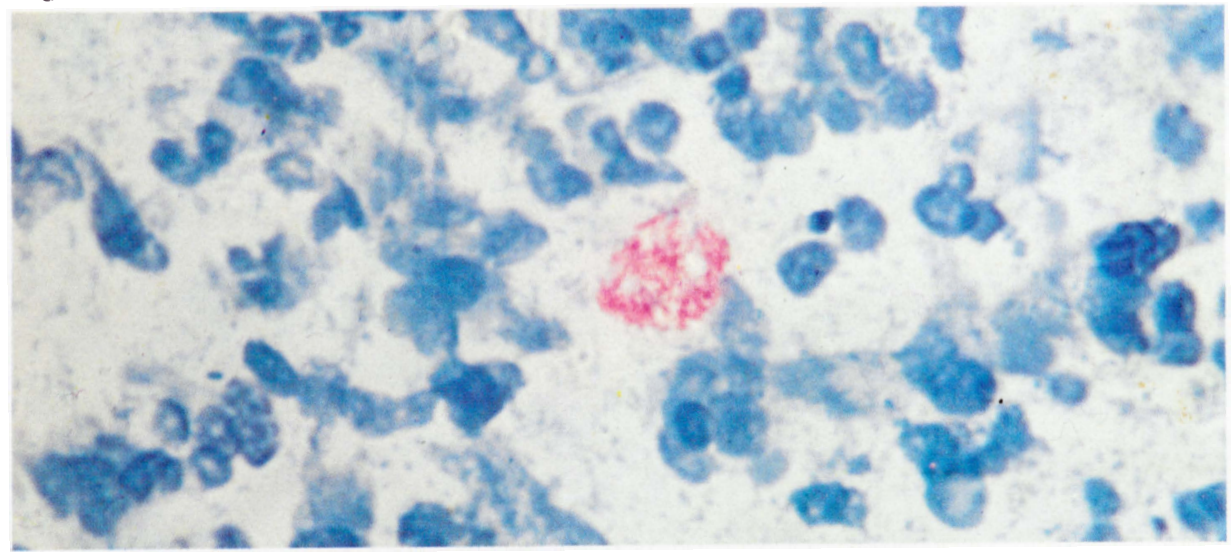

Fig. 3. Sneeze at $30 \mathrm{~cm}$. Large glohus in droplet (diameter $0.4 \mathrm{~mm}$ ).

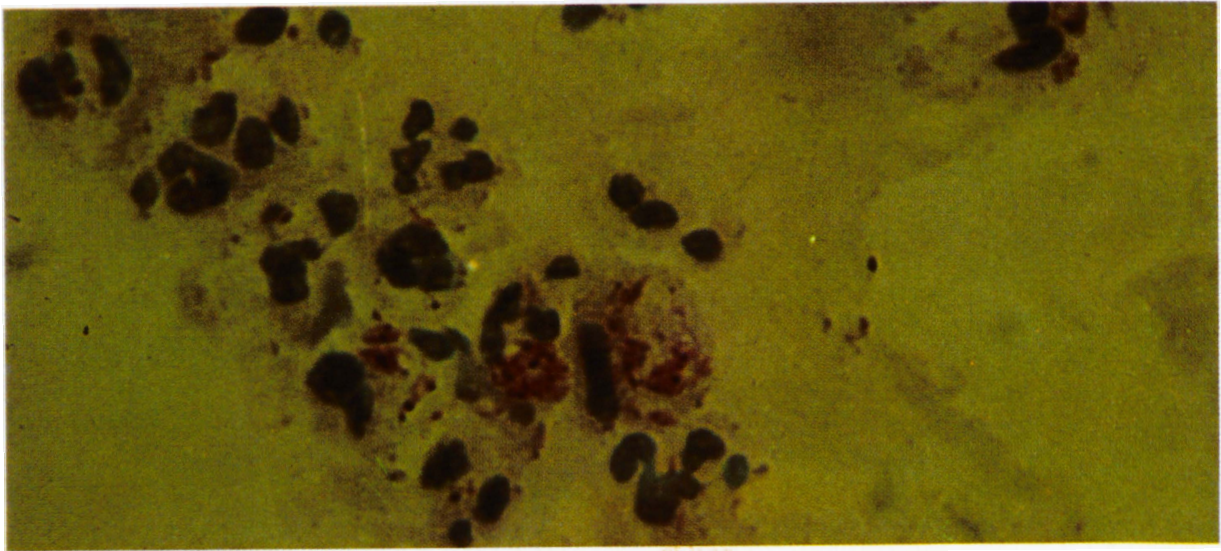

Fig. 4. Sneeze at $50 \mathrm{~cm}$. 
TABLE 1

Release of acid-fast bacilli from the nose and mouth

\begin{tabular}{lccccc}
\hline Experiment & $\begin{array}{c}\text { Number of } \\
\text { slides }\end{array}$ & $\begin{array}{c}\text { Distance } \\
\text { from face } \\
(\mathrm{cm})\end{array}$ & $\begin{array}{c}\text { Time } \\
(\mathrm{min})\end{array}$ & $\begin{array}{c}\text { Number of } \\
\text { droplets }\end{array}$ & AFB present \\
\hline Sneezing & 2 & 20 & & 500 & large numbers \\
Sneezing & 3 & 30 & & 340 & large numbers \\
Sneezing & 1 & 50 & 10 & 7 & numerous \\
Talking & 3 & 10 & 20 & 10 & nil \\
Talking & 2 & 5 & $?$ & nil & nil \\
Snoring & 3 & 10 & $?$ & nil & nil \\
Panting & 2 & 20 & $?$ & nil & nil \\
Panting & 2 & 5 & & & \\
\hline
\end{tabular}

\section{Discussion}

These findings confirm those of Schäffer where sneezing is concerned. In the case of untreated patients with severe lepromatous leprosy enormous numbers of acid-fast bacilli are projected from the nose during sneezing to distances at any rate up to $50 \mathrm{~cm}$ from the face, including bacilli morphologically normal. Taken in conjunction with Rees's findings on the viability of $M$. leprae outside the body (Davey and Rees, 1974) our findings strongly suggest that droplet infection could play a significant role in the transmission of leprosy.

It is probable that only patients with untreated lepromatous leprosy and heavy involvement of the nasal mucosa will yield positive findings in droplets, and it is surprising how non-apparent such cases can sometimes be (Pedley, 1970). It is most unlikely that borderline leprosy even in reaction is associated with the presence of acid-fast bacilli in the nasal secretions (Pedley, 1973). Enormous numbers are however found in the nasal discharge in untreated lepromatous leprosy at all stages (Davey and Rees, 1974), and this is the criterion for any selection of patients in the expansion of the studies reported here which is clearly desirable. Finally, it is probable that both of Schäffer's patients were also suffering from heavy involvement of the throat and larynx, and possibly of the palate as well, and this is likely to have contributed to his findings. It is a cause for thanksgiving that with the advent of present day treatment such advanced cases of lepromatous leprosy are rarely seen, and that with modern treatment bacilli can rapidly be eliminated from the nasal secretion.

\section{Acknowledgements}

Our grateful thanks are due to Dr R. J. W. Rees for much advice and encouragement. Thanks are also due to Dr D. J. Harman of the Leprosy Study Centre for the photomicrographs, and to the Leprosy Mission for covering the cost of colour photographs and for all their support without which this paper could not have been written. 


\section{References}

Davey, T. F. and Rees, R. J. W. (1974). The nasal discharge in leprosy: clinical and bacteriological aspects. Lepr. Rev. 45, 121.

Pedley, J. C. (1970). Composite skin contact smears: a method of demonstrating the non-emergence of $M$. leprae from intact lepromatous skin. Lepr. Rev. 41, 31.

Pedley, J. C. (1973). The nasal mucus in leprosy. Lepr. Rev. 44, 33.

Schäffer (1898). On the Spread of Leprosy Bacilli from the Upper Parts of the Respiratory Tract. Arch. Derm. Syph. XLIV, 159. 